\title{
Analysis of Critical values in NABL (National Accreditation Board for Testing and Calibration Laboratories) accredited Hematology and Clinical Pathology laboratory.
}

\author{
Killol Nathubhai Desai and Sanjay Chaudhari \\ 'Department of Pathology, GMERS Medical College, Junagadh, Gujarat, India \\ ${ }^{2}$ Department of pathology, Pramukhswami Medical College, Karamsad, Gujarat, India
}

\begin{abstract}
Background: Reporting of laboratory critical values has become an issue of national attention as illustrated by recent guidelines described in the National Patient Safety Goals of the Joint Commission on Accreditation of Healthcare Organizations. They may be considered an important laboratory outcome measurement because they reflect clinical effectiveness, patient safety and operational efficiency

Aims: To improve effectiveness, patient safety and operational efficiency by improving laboratory outcome measurement. Settings and Design: Cross-sectional study done at Shree Krishna Hospital, Karamsad, from January 2012 to December 2013.

Methods and Material: All data were obtained from reports generated by hematology and clinical pathology laboratory that has been recorded into critical call back log.

Statistical analysis used: The parameters were evaluated using descriptive statistical analysis with IBM Statistical Package for the Social Sciences v 20.0 and Microsoft Office Excel 2007 software.

Results: The hematology and clinical pathology laboratory reported 19,423 critical values. The majority of critical callbacks (78.4\%) resulted from testing performed in the hematology laboratory. The analytes most commonly called back were Hemoglobin and Urine Ketone. We have recorded maximum $52.7 \%$ call back from inpatient department followed by emergency department $34.2 \%$ and outpatient department $13.1 \%$. The mean time between entering value in the critical callback register and conveying the information to the patient location or ordering clinician was 21 minutes for IPD, 30 minutes for OPD and 20 minutes for ED.
\end{abstract}

Conclusions: "Every laboratory should have at its disposal a procedure to notify critical

Keywords: Critical call back, Turn around time, LIS (Laboratory information system)

\section{Introduction}

Critical value reporting originally was highlighted by Lundberg, who defined a critical value as a result suggesting that the patient is in imminent danger unless appropriate therapy is initiated promptly. ${ }^{[1]}$ In the 30 years since Lundberg's observations, the concept of defining critical values and systems for reporting have been adopted widely by laboratories throughout the world. ${ }^{[2]}$

In the United States, laboratory accrediting agencies such as the Joint Commission on Accreditation of Healthcare Organizations (JCAHO) and the College of American Pathologists have made critical value reporting part of the requirements for accreditation. ${ }^{[3,4]}$

The recent national focus on patient safety has brought increased attention to the issue of laboratory critical value reporting. The JCAHO has made improving the process of critical value reporting a National Patient Safety Goal for the years 2004 through 2006. ${ }^{[3]}$ The JCAHO requires health care organizations to track and improve the timeliness of reporting and receipt of critical test results by the responsible licensed caregiver. Moreover, the JCAHO has defined critical test results as not only laboratory tests but also imaging studies, electrocardiograms and other diagnostic studies. Therefore, the process of critical value reporting is of interest across the health care organizations.

Critical value reporting parameters may be considered an important laboratory outcome measurement because they reflect clinical effectiveness, patient safety and operational efficiency. For the critical value reporting process to be effective, the organization must understand and address the variables involved in the process. This information is not readily available in the literature. Most reports have analyzed only a few analytes for short periods or have reviewed a small number of critical values in a number of different institutions. ${ }^{[5,6,7]}$ In the present study, we analyzed 24 months of critical value data and 
19,423 individual critical results to understand the scope of critical value reporting and identify opportunities for process improvement.

\section{Materials and Methods:}

This prospective cross-sectional study was conducted at Central Diagnostic Laboratory, Hematology and Clinical pathology section, Shree Krishna Hospital from January 2012 to December 2013. All major medical and surgical specialities are supported by the hospital, along with pediatric and obstetric services and extensive primary care and specialty outpatient practices. From January 2012 to December 2013, the laboratories performed 90,000 reportable tests, of which $34 \%$ were for inpatients, $55 \%$ for outpatients and $11 \%$ for emergency department (ED) patients.

Critical Callback Procedures: Table 1 shows the critical callback list for hematology and clinical pathology laboratory that is in use at our institution. Responsible laboratory personnel (Resident Doctors, Lab Technologist) will ensure the validation of the result by repetition of test or by recalibration of parameter if necessary and/ or by checking quality control result. If the result is in the critical value (upper or lower) the lab personnel would communicate with the responsible caregiver [Consultant, Registrar, Medical Officer, Nurse and Operations associate (Clerical staff who perform clinical support function)] to inform the values over phone. At the same time he/she will also inform the result to the concerned laboratory doctor (consultant, senior doctor and incharge). For outpatient critical value would be informed to responsible consultant or his/her medical assistant (medical officer). The laboratory personnel will record details of the critical call back (parameter, critical value, informed person, date, time) in a register.

Data Collection and Analysis: All data were obtained from reports generated by hematology and clinical pathology laboratory that has been recorded into critical call back register.

\section{Statistical Analysis}

The obtained parameters were evaluated using descriptive statistical analysis. Statistical analyses were performed using the IBM SPSS (Statistical Package for the Social Sciences v 20.0) and Microsoft Office Excel 2007 software.

\section{Results}

During the period of the study, the hematology and clinical pathology laboratories reported 19,423 critical values. During the same period, these laboratories reported 90,000 test results. Therefore, tests with critical values represented approximately $21.6 \%$ of the total test results reported.

The majority of critical callbacks (78.4\%) resulted from testing performed in the hematology laboratory (Table 2). The clinical pathology laboratory accounted for $21.6 \%$ of critical callbacks. The analytes most commonly called back were hemoglobin (5212 results; $26.8 \%$ of critical results) and Urine Ketone (3100 results; $16 \%$ of critical results) (Table 3 ).

We have recorded maximum 10,242 (52.7\%) call back from inpatient department (IPD) followed by emergency department (ED) 6651 (34.2\%) and outpatient department (OPD) 2530 (13.1\%) (Table 4).

Critical value calls were prominent 11,577 (59.6\%) in morning shift and minimum $561(2.9 \%)$ in night shift (Table 5).

The "in-laboratory" turnaround time for each critical value was determined to assess the timeliness of critical value reporting. Turn around time is the time period between receiving of a sample and generation of report. ${ }^{[3]}$ For the 19,423 critical values, the mean time between entering value in the critical callback register and conveying the information to the patient location or ordering clinician was 21 minutes (12-30 minutes) for IPD, 30 minutes (1545 minutes) for OPD and 20 minutes (10-30 minutes) for ED (Table 6). Delay in critical value reporting correlated with testing performed on outpatients and testing ordered on requisitions lacking the name of the ordering clinician or the ordering location. Tests performed in settings where there is continuous technologist presence (e.g. Coagulation study) were called back faster than tests performed in other areas. This information was useful as we began to implement measures to improve critical value reporting in all areas of the laboratory.

Table 1: List of Critical values in hematology and clinical pathology laboratories.

\begin{tabular}{|c|c|c|c|}
\hline SL. NO & Discipline & Test parameter & $<$ Hemoglobin \\
\hline 1. & Hematology & Platelet & $<5.0 \mathrm{gm} / \mathrm{dl}$ \\
\hline 2. & Hematology & INR (International normalised ratio) & $<50000 / \mathrm{uL}$ \\
\hline 3. & Hematology & Total leucocyte count & $>5.0$ \\
\hline 4. & Hematology & & $>20000 / \mathrm{cmm}<4000 / \mathrm{cmm}$ \\
\hline
\end{tabular}




\begin{tabular}{|c|c|c|c|}
\hline 5. & Hematology & Absolute neutrophils count & $<1800 / \mathrm{cmm}$ \\
\hline 6. & Hematology & Immature: Total neutrophils ratio & $>0.2$ (Pediatric patients) \\
\hline 7. & Hematology & Malaria parasite & Present \\
\hline 8. & Hematology & Malaria antigen & Positive \\
\hline 9. & Hematology & Blast cells & Positive \\
\hline 10. & Clinical Pathology & Urine Ketone & $>5$ cells \\
\hline 11. & Clinical Pathology & CSF- cell count & \\
\hline
\end{tabular}

Table 2: Evaluation of Critical calls back in hematology and clinical pathology laboratories.

\begin{tabular}{|c|c|c|c|}
\hline Laboratory & Hematology & Clinical pathology & Total \\
\hline Critical calls back & $15,223(78.4 \%)$ & $4200(21.6 \%)$ & 19,423 \\
\hline
\end{tabular}

Table 3: Evaluation of Critical values by parameters

\begin{tabular}{|c|c|}
\hline \multicolumn{1}{|c|}{ Test parameters } & No. Of Critical calls back \\
\hline Hemoglobin & $5212(26.8 \%)$ \\
\hline Platelet & $2022(10.4 \%)$ \\
\hline INR (International normalised ratio) & $1958(10.1 \%)$ \\
\hline Total leucocyte count & $3331(17.1 \%)$ \\
\hline Absolute neutrophils count & $521(2.7 \%)$ \\
\hline Immature: Total neutrophils ratio & $412(2.1 \%)$ \\
\hline Malaria parasite & $1556(8 \%)$ \\
\hline Malaria antigen & $148(0.8 \%)$ \\
\hline Blast cells & $63(0.3 \%)$ \\
\hline Urine Ketone & $3100(16.0 \%)$ \\
\hline CSF- cell count & $1100(5.7 \%)$ \\
\hline Total & 19,423 \\
\hline
\end{tabular}

Table 4: Evaluation of critical values by clinical care areas

Clinical care areas

Inpatient department (IPD)

Outpatients department (OPD)

Emergency department (ED)

Total
No. Of Critical calls back

$10,242(52.7 \%)$
$2530(13.1 \%)$
$6651(34.2 \%)$
19,423

Table 5: Frequency of Critical Calls back in each shift.

\begin{tabular}{|c|c|c|c|c|c|}
\hline Timing & Early morning & Afternoon & Evening & \multicolumn{2}{|c|}{ Night } \\
\hline No. Of Critical calls back & $11,577(59.6 \%)$ & $4566(23.5 \%)$ & $2719(14 \%)$ & $561(2.9 \%)$ & 19,423 \\
\hline
\end{tabular}

Table 6: Evaluation of Critical values by TAT (Turn Around Time)

\begin{tabular}{|l|c|c|c|}
\hline \multirow{2}{*}{ Clinical care areas } & \multicolumn{3}{|c|}{ TAT (Turn Around Time) (Minutes) } \\
\cline { 2 - 4 } & Minimum & Maximum & 21 \\
\hline Inpatient department (IPD) & 12 & 30 & 30 \\
\hline Outpatients department (OPD) & 15 & 45 & 20 \\
\hline Emergency department (ED) & 10 & 30 & \\
\hline
\end{tabular}




\section{Discussion:}

In this study, we provide a comprehensive view of the critical value reporting process in a large medical center. We provide details regarding the scope, volume, timing and operational aspects of critical value reporting. Many of these parameters should be applicable to a variety of settings. This analysis provides a context for comparison and process improvement.

Increasing workload in the clinical laboratory makes it important to achieve efficient use of laboratory resources to maximize clinical benefits. Expansion of critical callback lists to include testing that does not meet the criterion of the "imminent danger" standard may dilute the urgency of a critical value call and lead to unnecessary interruptions for clinicians. For example, critical value calls for high INR levels will not be of clinical value for patients receiving heparin in cardiac operations. In addition, there are many clinical settings (chemotherapy, malignancy) in which the "critical" test result is expected and reporting of this value may not contribute to improved patient care.

Communication by telephone, especially when performed by technologists, is a costly practice in terms of the resources required to complete the phone calls and document the process. For this reason, it is helpful to try to reduce the number of phone calls by careful review of the critical values list. In addition to determining which tests are to be included in the critical values list, another important strategy is to examine the consequences of changing the boundaries for critical value reporting. These boundaries must be defined in consultation with clinicians. Small changes in critical value reporting parameters may result in the addition or loss of thousands of phone calls for the laboratory staff.

Outpatient critical values present unique challenges in timely reporting to clinicians. One of the strongest correlates of delayed reporting of critical values was the samples being obtained from an outpatient. Outpatient critical values are challenging to communicate to the responsible clinician because there often are different approaches in various practices for determining patient coverage. Unlike inpatients, there is no fixed patient location that can be phoned.

Another factor we identified as causing delay for outpatients was illegible or missing patient information. We have noted that recent improvements in the critical value communication times have coincided with increased awareness of critical value monitoring. We presently are working with our outpatient practices to improve communication between the laboratories and the outpatient care centers.
Another contributor to delay in outpatient critical value reporting is the heterogeneity of the outpatient population, with specimens arriving from health centers, clinics, urgent care centers, dialysis centers, and physicians' offices. Each of these areas is likely to have a different call schedule, answering service and cross-coverage procedure, making reliable communication with the responsible licensed caregiver challenging. The nature of outpatient specimen transport and processing often results in outpatient test results being generated in the evening when the outpatient clinic or physician's office is closed. The laboratory must have a mechanism to determine on-call coverage and work with outpatient practices to improve the communication processes.

The potential for technological solutions to improve the process of critical value reporting is evident in numerous reports. ${ }^{[8,9]}$ The use of information technology to automatically communicate with the responsible provider has been demonstrated to reduce the critical value reporting time in controlled settings. For implementation of automated critical value reporting, interfaces from the LIS to technologies that facilitate bidirectional communication (such as e-mail or 2 - way pagers) need to be developed. An important component in such a system is the ability of the automatic reporting system to reliably determine the identity of the responsible provider. At larger medical centers, this task can be challenging because there may be different coverage lists, tests ordered by consultants unknown to the primary caregiver and patient transfers to different locations. An electronic reporting system potentially could create dangerous delays in communication if not properly implemented. The system needs to have an "acknowledgment" function such that the laboratory can ensure that the responsible caregiver received the result. ${ }^{[10]}$ Electronic systems also require an escalation procedure so that lack of acknowledgment of the critical result prompts an alternative approach for communication.

Development of LIS middleware with alert reporting software should permit highly nuanced approaches to critical value reporting in the near future. Rules-based logic can be applied to laboratory values to build alerts that take into account not only the result value, but also other related results, a change in the current test result from previous results (delta checks), patient demographics, ordering provider and other parameters to customize the alerting to the patient's condition and the needs of the clinical team for notification. For example, many oncology physicians do not want to be notified regarding patients with neutropenia. The ability to provide a physician specific critical values list could eliminate a large number of unnecessary critical value calls. These systems, when interfaced 
with automated alerting systems, will have the potential to improve patient safety and provide more context-sensitive critical value reporting. At present, practical implementation of this scenario would be constrained by regulations (particularly the JCAHO National Patient Safety Goals) that require all critical results to be communicated and do not allow for more nuanced approaches.

\section{Conclusion}

"Every laboratory should have at its disposal a procedure to notify critical results. A consensus should be reached with clinicians to establish a specific list of critical limits according to the type of patient and the timeliness of laboratory test". We are proud to introduce the system of critical call back system in our laboratory which is NABL accredited.

\section{References:}

1. Lundberg GD. When to panic over abnormal values. Med Lab Obs 1972;4:47-54.

2. Lundberg GD. Critical (panic) value notification: an established laboratory practice policy (parameter). JAMA 1990;263:709.

3. Joint Commission on the Accreditation of Healthcare Organizations: National Patient Safety Goals. Available at: http://www.jcaho.org/accredited+organizations/patient+safety/ npsg.htm. Accessed June 22, 2014.

4. Center for Medicare and Medicaid Services. Department of Health and Human Services. Clinical Laboratory Improvement Amendments of 1988: 68 Federal Register (2003) (codified at 42 CFR 493.1291(g)).1047

5. Lum G. Assessment of a critical limit protocol for point-of care glucose testing. Am J Clin Pathol 1996;106:390-395.

6. Howanitz PJ, Steindel SJ, Heard NV. Laboratory critical values policies and procedures: a College of American Pathologists Q-Probes study in 623 institutions. Arch Pathol Lab Med 2002;126:663-669.

7. Kuperman GJ, Boyle D, Jha A, Rittenberg E, Ma'Luf N, Tanasijevic MJ et al. How promptly are inpatients treated for critical laboratory results? J Am Med Inform Assoc 1998;5:112-119.

8. Bates DW, Pappius E, Kuperman GJ, Sittig D, Burstin H, Fairchild D et al. Using information systems to measure and improve quality. Int J Med Inform 1999;53:115-124.

9. Tate KE, Gardner RM, Weaver LK. A computerized laboratory alerting system. MD Comput 1990;7:296-301.

10. Dighe AS, Rao A, Coakley AB, Lewandrowski KB. Analysis of laboratory critical value reporting at a large academic medical center. AM J Clin Pathol 2006;125:758-764.

*Corresponding author:

Dr. Killol Nathubhai Desai, A-301, Staff Quarters, GMERS Medical College, Paddock road, Junagadh, Gujarat, India. Pin: 362001, Phone: +91 09428050253

Email: drkilloldesai@gmail.com 